\title{
Spicaticribra kingstonii Johansen, Kociolek \& Lowe (Thalassiosirales, Bacillariophyta): primera cita del género y de la especie para las aguas continentales de Chile
}

\author{
Spicaticribra kingstonii Johansen, Kociolek \& Lowe (Thalassiosirales, Bacillariophyta): \\ first report of the genus and species from Chilean inland waters
}

\author{
Patricio Rivera \& Fabiola Cruces \\ Departamento de Botánica, Universidad de Concepción, Casilla 160-C, Concepción, Chile. \\ *privera@udec.cl
}

\begin{abstract}
RESUMEN
Spicaticribra kingstonii Johansen, Kociolek \& Lowe fue hallada en aguas de la Laguna Grande de San Pedro, Chile. Esta es la primera cita del género y de la especie para el país. Los individuos chilenos carecen de una fultoportula central en la cara valvar, de tubos externos en las fultoportulae marginales y poseen aréolas con cribra internas anastomosadas en forma de espiga, características que concuerdan con las del género Spicaticribra. En el presente trabajo se entrega una diagnosis con las características relevantes de los individuos chilenos, se comenta la variabilidad de algunas de ellas, se menciona su relación con taxones afines, y fotografías obtenidas mediante técnicas de microscopia fotónica y electrónica de barrido ilustran esos caracteres.
\end{abstract}

Palabras clave: Primera cita, diatomea, Laguna Grande, Chile.

\begin{abstract}
Spicaticribra kingstonii Johansen, Kociolek \& Lowe was found in Laguna Grande de San Pedro, Chile. This is the first report of the genus and species for the country. The Chilean specimens lack of a central fultoportula on the valve face, as well as of external extensions of the marginal fultoportulae, and have areolae with internal spicate anastomosing cribra, features present in the genus Spicaticribra. In this paper we describe the characteristics of the Chilean cells, comments on the variability of some of its morphological features and about its relationship with other closely related taxa, and light and scanning electron microscope photographs aim to provide information about those features.
\end{abstract}

KeYwords: First report, diatom, Laguna Grande, Chile.

\section{INTRODUCCIÓN}

El género Spicaticribra, y la especie tipo Spicaticribra kingstonii (Thalassiosirales), fueron descritos por Johansen, Kociolek \& Lowe en el año 2008 a partir de material recolectado en el Lago Fontana, Carolina del Norte, USA. El género fue caracterizado por poseer cribra internas semicontinuas en forma de espiga, por carecer de una fultoportula central sobre la cara valvar y de extensiones externas de las fultoportulae marginales. En 2012, Khursevich \& Kociolek agregan las siguientes características propias del género: valvas planas o dobladas, una a varias rimoportulae en el manto valvar, alargadas internamente y con o sin tubo externo, y fultoportulae con 2-4 poros satélites con o sin tubo externo dispuestas en el manto valvar (raramente sobre la cara valvar). Sobre esta base, los autores mencionados propusieron trasladar a Spicaticribra todas las especies que presenten aréolas con cribra internas continuas y foramen externo, como son diversas especies incluidas en Thalassiosira Cleve y en Conticribra Stachura-Suchoples \& Williams (2009), género que también posee una morfología general similar a Spicaticribra.

Hasta la fecha se conocen cerca de ocho especies pertenecientes al género Spicaticribra, fósiles o recientes (Khursevich \& Kociolek 2012, Johansen et al. 2008) distribuidas en Norteamérica, Europa, Asia y Sudamérica, número que debería aumentar si se revisa la morfología de 
otras especies, especialmente del género Thalassiosira.

Análisis rutinarios del fitoplancton de la Laguna Grande de San Pedro, VIII Región, Chile, permitieron reconocer la presencia de una diatomea de contorno circular, muy silicificada, y con valvas provistas de un área central notoriamente diferente al resto de la cara valvar. La observación de estos individuos mediante técnicas de microscopía fotónica y electrónica de barrido puso en evidencia los caracteres morfológicos que tipifican a Spicaticribra kingstonii Johansen, Kociolek \& Lowe, género y especie no señalados anteriormente para el país.

\section{MATERIALES Y MÉTODOS}

La Laguna Grande de San Pedro (36 $51^{\prime}$ S, $73^{\circ} 06^{\prime} \mathrm{W}$ ) presenta una marcada influencia mediterránea, donde las precipitaciones se concentran en invierno con una temperatura media anual de $18,1^{\circ} \mathrm{C}$ (Fuenzalida 1971). El cuerpo de agua se encuentra a 16 m.s.n.m., posee una superficie de 1,55 $\mathrm{km}^{2}$ y una profundidad máxima de 13,5 $\mathrm{m}$. La columna de agua presenta una temperatura máxima de $24^{\circ} \mathrm{C}$ y una mínima de $12,2^{\circ} \mathrm{C}, \mathrm{pH}$ de 7,0 , conductividad de $84,1 \mu \mathrm{S} / \mathrm{cm}$, oxígeno disuelto de $8,8 \mathrm{mg} / 1$, transparencia de 3,7 $\mathrm{m}$ y una concentración de fósforo y nitrógeno total de 11,9 y $0,225 \mathrm{mg} / 1$ respectivamente (Parra et al. 2003). El material estudiado fue recolectado el 23 de mayo de 2012 con red de $20 \mu \mathrm{m}$ de trama y se encuentra depositado en la Colección Diatomológica de la Universidad de Concepción con los números DIAT-CONC M-3436, DIAT-CONC 7497, 7498. Luego de la eliminación de la materia orgánica según el método de Battarbee (1986), alrededor de cincuenta células fueron observadas mediante microscopía óptica (MO, Olympus CX-31) y electrónica de barrido (MEB, Jeol JSM-6380LVY). La terminología usada corresponde a la sugerida por Anonymous (1975), Ross et al. (1979) y Håkansson (2002).

\section{RESULTADOS}

Spicaticribra kingstonii Johansen, Kociolek \& Lowe Figs. 1-2.

Johansen et al., Diatom Research 23(2): 368-369, Figs. 1-24, 2008.

Thalassiosira rudis Tremarin, Ludwig, Becker \& Torgan in Luwdig et al., Diatom Research 23(2): 391, Figs. 1-57, 2008.

Spicaticribra rudis (Tremarin, Ludwig, Becker \& Torgan) Tuji, Leelahakriengkrai \& Peerapornpisal, Memoirs of the National Museum of Nature and Science, Tokyo, 48: 146, 2012.
Células iniciales circulares, 25-28 $\mu \mathrm{m}$ de diámetro, con la superficie convexa y con aréolas orientadas en líneas radiales algo desordenadas, de mayor tamaño hacia el margen (Fig. 1 A). Frústulos cilíndricos, muy silicificados, 13-25 $\mu \mathrm{m}$ de diámetro (Figs. $1 \mathrm{E}, 2 \mathrm{~A}$ ). Cingulum formado por 5 bandas abiertas que disminuyen progresivamente en su ancho hacia el lado abvalvar (Fig. 1 B), provistas de un septo interno en el lado advalvar (Fig. 1 D), de una notoria lígula (Fig. 1 B) y una pequeña antilígula (Fig. $1 \mathrm{C}$ ); la valvocópula lleva una hilera de poros, 7-8 en $1 \mu \mathrm{m}$ (Fig. $1 \mathrm{C}$ ). Células vegetativas con la cara valvar plana (Figs. 1 F-G, 2 C-D) y manto inclinado (Fig. 2 A-B) que termina en un notorio borde ornamentado externamente con delgados surcos orientados en el sentido del eje pervalvar, 5-6 en $1 \mu \mathrm{m}$ (Fig. 1 B). Aréolas dispuestas en estrías radiales, rectas (Fig. 1 F-G), 18-19 (21) en $10 \mu \mathrm{m}$, y 18-19 (21) aréolas en $10 \mu \mathrm{m}$ en cada estría, externamente las aréolas centrales son de mayor tamaño e irregulares en forma y distribución (Figs. 1 F-G, 2 A-B). Internamente las aréolas poseen cribra delicadas y anastomosadas en forma de espiga que cubren tanto la cara valvar como el manto (Fig. $2 \mathrm{D})$, donde se observa continua y formada por diminutos poros redondeados (Fig. 2 G). Fultoportulae presentes sólo en el manto valvar (Fig. 2 C-D), 4-5 en $10 \mu \mathrm{m}$; externamente presentan sólo una abertura circular y cónica, algo elevada sobre la valva (Figs. 1 C, 2 B), internamente son largos tubos con 3 poros satélites, cada uno rodeado por un alto y prominente recubrimiento silíceo unido al tubo central (cobertor) (Fig. 2 E-I). Hay 1-2 rimoportulae ubicadas muy cerca del anillo marginal de fultoportulae (Fig. 2 C-D), pero más próximos al borde valvar (Fig. 2 F, H-I); externamente carecen de tubo, internamente son tan largos como las fultoportulae, algo doblados y con los labios ensanchados (Fig. 2 F, H-I).

\section{DISCUSIÓN}

Las características del material estudiado coinciden exactamente con la descripción de Spicaticribra kingstonii, donde la presencia de cribra internas anastomosadas y en forma de espiga, fultoportulae ausentes en la cara valvar y fultoportulae marginales carentes de tubo externo, parecen ser las características más relevantes. Para la palabra inglesa "cowling", que de acuerdo con Theriot \& Serieyssol (1994), Hayashi et al. (2007) y Prasad \& Nienov (2011) corresponde a un reborde silíceo que envuelve al poro satélite y está unido al tubo interno de la fultoportula, se propone la palabra española "cobertor", del latín coopertorium, que según el Diccionario de la Real Academia Española (22a edición) significa cubierta, manta. Algunas valvas exhiben gránulos silíceos redondeados ubicados principalmente sobre el manto valvar, cuya densidad es variable en los individuos (Fig. 2 A-B), característica no presente en el 

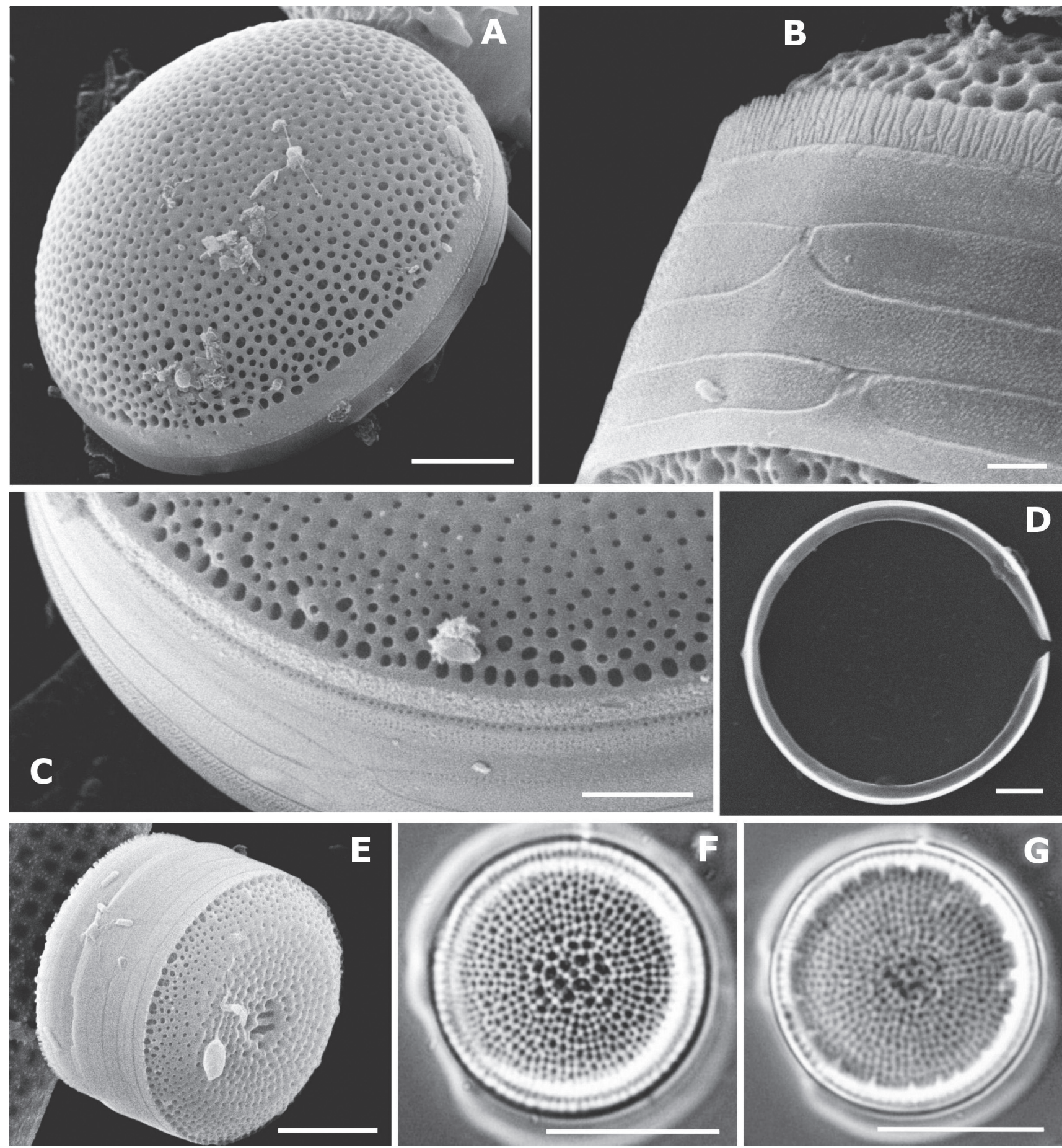

Figura 1. Spicaticribra kingstonii. A-E, Microscopía Electrónica de Barrido; F-G, Microscopía Optica. A. Vista externa de una célula inicial. B-C. Cingulum con bandas abiertas, lígula notoria; valvocópula con una hilera de poros en lado advalvar. Borde valvar con surcos. D. Banda abierta, septada. 'E. Centro de la valva levemente hundido. F-G. Vista externa e interna de valvas con estrías radiales, aréolas centrales de mayor tamaño e irregulares en forma y distribución, y fultoportulae marginales. Escalas: $\mathrm{A}, \mathrm{E}=5 \mu \mathrm{m} ; \mathrm{B}=1 \mu \mathrm{m} ; \mathrm{C}-\mathrm{D}=2 \mu \mathrm{m}$; $\mathrm{F}-\mathrm{G}=10 \mu \mathrm{m}$.

Figure 1. Spicaticribra kingstonii. A-E, Scanning Electron Microscopy; F-G, Light Microscopy. A. Domed initial cell valve, external view. B-C. Cingulum with open bands, prominent ligulae; valvocopula with a row of pores at the advalvar side. Mantle edge grooved D. Septate open band. E. Valve center slightly depressed. F-G. External and internal views of valves with radial striae, central areolae larger than others and irregular in shape and distribution, and marginal fultoportulae. Scale bars: A, E $=5 \mu \mathrm{m} ; \mathrm{B}=1 \mu \mathrm{m}: \mathrm{C}-\mathrm{D}=2 \mu \mathrm{m} ; \mathrm{F}-\mathrm{G}=10 \mu \mathrm{m}$. 

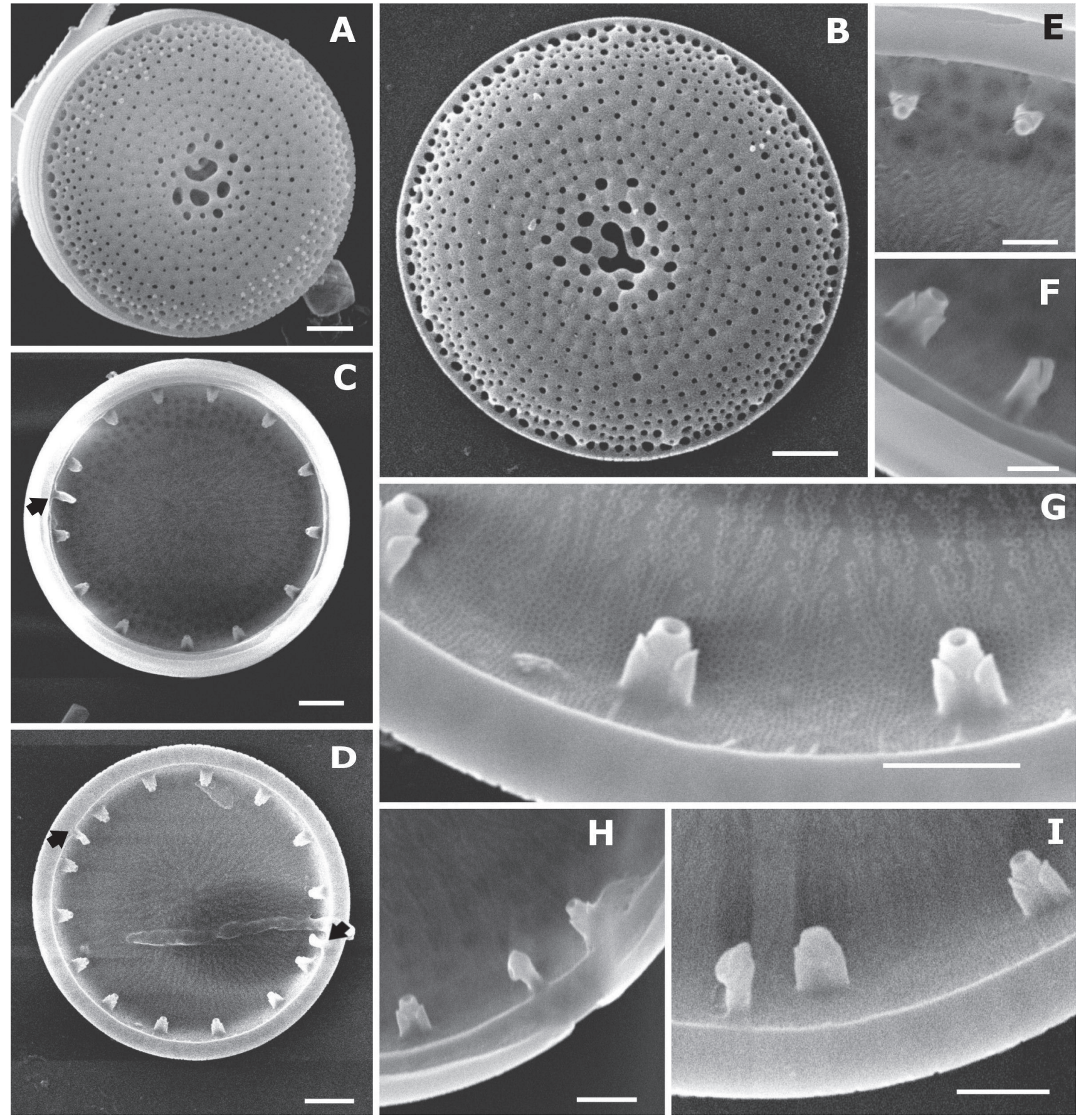

Figura 2. Spicaticribra kingstonii con Microscopía Electrónica de Barrido. A-B. Aréolas centrales de mayor tamaño e irregulares en forma y distribución. Manto valvar con y sin gránulos silíceos. Aberturas externas de las fultoportulae redondeadas, algo elevadas sobre la valva. C-D. Vista interna de valvas con una y dos rimoportulae marginales (flechas). E. Fultoportulae con tres poros satélites. F. Rimoportula de igual largo que las fultoportulae. G. Cribra interna anastomosada en forma de espiga. Fultoportula con cobertores altos. H-I. Rimoportulae con el extremo interno ensanchado, ubicadas más cerca del margen que las fultoportulae. Escalas: A-D = $2 \mu \mathrm{m} ; \mathrm{E}, \mathrm{G}-\mathrm{I}=1 \mu \mathrm{m} ; \mathrm{F}=0.5 \mu \mathrm{m}$.

FiguRE 2. Spicaticribra kingstonii in Scanning Electron Microscopy. A-B. Central areolae much larger than others and irregular in shape and distribution. Valve mantle with or without siliceous granules. Raised rounded external pores of the fultoportulae. C-D. Internal view of valves with one or two marginal rimoportula (arrows). E. Fultoportulae with three satellite pores. F. Rimoportula with a similar length of fultoportulae. G. Spicate anastomosing internal cribra. Fultoportulae with tall cowlings. H-I. Rimoportulae with the internal end swollen, located closer to the valve edge than the fultoportulae. Scale bars: A-D $=2 \mu \mathrm{m} ; \mathrm{E}, \mathrm{G}-\mathrm{I}=1 \mu \mathrm{m} ; \mathrm{F}=0.5 \mu \mathrm{m}$. 
material tipo y que posiblemente depende de las condiciones ambientales. Al observar con microscopía electrónica de barrido frústulos en vista conectival del material recolectado en Chile, es posible notar que el centro de la valva está levemente hundido (Fig. 1 E), atributo no evidente al mirar frontalmente la cara valvar externa o interna (Figs. $1 \mathrm{~F}-\mathrm{G}$, 2 C-D), característica que sumada a la presencia de bandas septadas en el cingulum no fueron mencionadas en la descripción original de $S$. kingstonii, aunque en las figuras 18 y 23 de Johansen et al. (2008) se pueden reconocer las bandas septadas. Tampoco se mencionó en la descripción de esta especie la posición más marginal que toman las rimoportulae con respecto a las fultoportulae (evidente en la Fig. 21 de Johansen et al. 2008).

Thalassiosira rudis Tremarin, Ludwig, Becker \& Torgan (in Ludwig et al. 2008) fue descrita a partir de material recolectado en varios ecosistemas dulceacuícolas de Brasil. La especie se caracteriza por poseer un cingulum estructurado por bandas abiertas y septadas, aréolas centrales de mayor tamaño que las restantes, provistas de cribra internas anastomosadas y ramificadas dicotómicamente, 1-3 rimoportulae situadas más cerca del margen valvar que las fultoportulae, ausencia de una fultoportula central pero formando un anillo marginal, cada uno provisto con tres poros satélites. Además, los autores indican que las fultoportulae marginales presentan un tubo exterior corto; sin embargo las figuras 30 y 36 de ese trabajo muestran solamente la apertura externa de las fultoportulae que son levemente engrosadas, y no un tubo propiamente tal. En la diagnosis latina Tremarin et al. describen la cara valvar externa como convexa y levemente hundida en el centro. Si bien lo segundo es verdadero y también fue visto en el material chileno estudiado, las valvas vegetativas no son convexas sino planas, con el manto valvar inclinado, y solamente la superficie de las células iniciales en esta especie son fuertemente convexas. Considerando que los caracteres que definen a Thalassiosira rudis son exactamente iguales a aquellos que están presentes en Spicaticribra kingstonii, T. rudis debe ser considerada como un sinónimo de esta última especie.

Tuji et al. (2012) luego de estudiar células muy parecidas a Spicaticribra kingstonii y a Thalassiosira rudis, recolectadas en Japón y Tailandia, concluyen que los individuos de aguas japonesas, por poseer aréolas centrales irregulares y rimoportulae alejadas del margen valvar, corresponden a Spicaticribra kingstonii, mientras que áquellos provenientes de Tailandia, con rimoportulae alargadas y curvadas, ubicadas en el margen valvar, y aréolas centrales más regulares corresponderían a Thalassiosira rudis, creando la nueva combinación Spicaticribra rudis (Tremarin, Ludwig, Becker \& Torgan) Tuji, Leelahakriengkrai \& Peerapornpisal. Sin embargo, y considerando la irregularidad de las aréolas centrales, Johansen et al. (2008) las describen para S. kingstonii como "contorno redondeado a irregular, con la abertura externa de mayor tamaño que las restantes"; por su parte Ludwig et al. (2008) comentan que en Thalassiosira, rudis son más grandes, de contorno irregular y dispuestas en forma irregular. Es decir, no hay diferencias significativas entre ambos taxones con respecto a esta característica. Con relación a la forma y posición de las rimoportulae, Johansen et al. (2008) las describen en S. kingstonii como alargadas, con el extremo ensanchado, ubicadas en el anillo de las fultoportulae. Sin embargo, la Fig. 24 de ese trabajo muestra una rimoportula ubicada más cerca del margen valvar que las fultoportulae. En Thalassiosira rudis, las rimoportulae fueron descritas como ubicadas "entre dos fultoportulae marginales, casi siempre equidistante de ambos" (Figs. 21-31, Ludwig et al. 2008), pero luego los autores comentan "rimoportula son alargadas, ubicadas fuera del anillo marginal de fultoportulae", y así aparece en la Fig. 35 de esa publicación. Es decir, las rimoportulae en ambas especies están desplazadas más hacia el margen valvar que las fultoportulae, pero no en el margen mismo, y tienen la misma forma y tamaño. De esta forma, Spicaticribra rudis (Tremarin, Ludwig, Becker \& Torgan) Tuji, Leelahakriengkrai \& Peerapornpisal debe ser considerada como otro sinónimo de $S$. kingstonii.

Aparte de la localidad chilena aquí descrita, Spicaticribra kingstonii ha sido encontrada en el Lago Fontana, U.S.A., localidad tipo (Johansen et al. 2008), en Tailandia como $S$. rudis y en diversos embalses de Japón como Spicaticribra kingstonii (Tuji et al. 2012), en el Lago Ikeda, Japón, como S. kingstonii (Tanaka \& Naguno 2009), en el Lago Fukugami, Japón, como S. kingstonii (Tanaka 2010), y en diversos ecosistemas continentales de Brasil, como Thalassiosira rudis (Ludwig et al. 2008).

De acuerdo con análisis moleculares usando $18 \mathrm{~S}$ rDNA , S. kingstonii presenta similitud con Cyclotella meneghiniana, aun cuando la similitud morfológica entre estas dos especies es muy baja (Tuji et al. 2012).

\section{AGRADECIMIENTOS}

Agradecemos al Dr. Luc Ector (Public Research CentreGabriel Lippmann, Luxembourg) por su constante apoyo y sugerencias durante la realización de este trabajo. También agradecemos a la Dra. Silvia E. Sala y a un segundo revisor (anónimo) por sus valiosas sugerencias y comentarios al texto, y al personal del Centro de Espectroscopía y Microscopía Electrónica de la Universidad de Concepción por su ayuda con el microscopio electrónico de barrido.

\section{BIBLIOGRAFÍA}

Anonymous. 1975. Proposal for a standardization of diatom terminology and diagnosis. Nova Hedwigia Beiheft 53: 
323-354.

Battarbee, R. 1986. Diatom analysis. In: B.E. Berlund (ed.), Handbook of Holocene Palaeoecology and Palaeohydrology, pp. 527-569. John Wiley \& Sons, Chichester,

Fuenzalida, H. 1971. Climatología de Chile. Publicación Interna Facultad de Ciencias Físicas y Matemáticas, Universidad de Chile. 73 pp.

HÁKAnsson, H. 2002. A compilation and evaluation of species in the genera Stephanodiscus, Cyclostephanos and Cyclotella with a new genus in the family Stephanodiscaceae. Diatom Research 17: 1-139.

Hayashi, T., Y. TAnimura \& H. SAKa. 2007. A fossil freshwater Thalassiosira, T. islandica sp. nov. (Bacillariophyta), with semicontinuous cribra and elongated marginal fultoportulae. Phycologia 46(4): 353-362.

Johansen, J., P. Kociolek \& R. Lowe. 2008. Spicaticribra kingstonii gen. nov. et sp. nov. (Thalassiosirales, Bacillariophyta) from Great Smoky Mountains National Park. U.S.A. Diatom Research 23(2): 367-375.

Khursevich, G. \& P. Kociolek. 2012. A preliminary, worldwide inventory of the extinct, freshwater fossil diatoms from the orders Thalassiosirales, Stephanodiscales, Paraliales, Aulacoseirales, Melosirales, Coscinodiscales and Biddulphiales. Nova Hedwigia Beiheft 141: 315-364.

Ludwig, T., P. Tremarin, V. Becker \& L. Torgan. 2008. Thalassiosira rudis sp. nov. (Coscinodiscophyceae): A new freshwater species. Diatom Research 23(2): 389-400.

Parra, O., C. Valdovinos, R. Urrutia, M. Cisternas, E. Habit \& M. Mardones. 2003. Caracterización y tendencias tróficas de cinco lagos costeros de Chile Central. Limnetica 22(1-
2): $51-83$.

Prasad, A. \& J. Nienov. 2011. Livingstonia (Thalassiosirales, Bacillariophyta), a new genus of fultoportulate centric diatoms from an Atlantic coastal plain river in Florida, southeastern United States. Phycologia 50(3): 264-280.

Ross, R., E.J. Cox, N.I. Karayeva, D.G. Mann, T.B.B. Paddock, R. Simonsen \& P.A. Sims. 1979. An amended terminology for the siliceous components of diatom cell. Nova Hedwigia Beiheft 64: 513-533.

Stachura-Suchoples, K. \& D. Williams. 2009. Description of Conticribra tricircularis, a new genus and species of Thalassiosirales, with a discussion on its relationship to other continuous cribra species of Thalassiosira Cleve (Bacillariophyta) and its freshwater origin. European Journal of Phycology 44(4): 477-486.

TANAKA, H. 2010. Spicaticribra kingstonii Johansen, Kociolek et Lowe found from Lake Fukugami, Okinawa Prefecture. Diatom 26: 44-45.

TANAKA, H. \& T. NAguno. 2009. First report of Spicaticribra kingstonii Johansen, Kociolek et Lowe with accompanying centric diatoms found from Lake Ikeda, Japan (Bacillariophyta). Japanese Journal of Phycology (Sorui) 57: 86-92.

Theriot, E. \& K. Serieyssol. 1994. Phylogenetic systematics as a guide to understanding features and potential morphological characters of the centric diatom family Thalassiosiraceae. Diatom Research 9(2): 429-450.

Tuji, A., P. Leelahakriengkrai \& Y. Peerapornpisal. 2012. Distribution and Phylogeny of Spicaticribra kingstonii rudis Species Complex. Memoirs of the National Museum of Nature and Science, Tokyo 48: 139-148.

Recibido: 23.04 .13

Aceptado: 05.08.13 